\title{
Residual amplitude modulation in interferometric gravitational wave detectors
}

\author{
Keiko Kokeyama, ${ }^{1, *}$ Kiwamu Izumi ${ }^{2,3}$ William Z. Korth, ${ }^{4}$ Nicolas Smith-Lefebvre, ${ }^{4}$ \\ Koji Arai, ${ }^{4}$ and Rana X. Adhikari ${ }^{4}$ \\ ${ }^{1}$ Louisiana State University, Physics and Astronomy, 202 Nicholson Hall, Baton Rouge, Louisianna 70806, USA \\ ${ }^{2}$ University of Tokyo, Department of Astronomy, 7-3-1 Hongo Bunkyo-ku, Tokyo 113-0033, Japan \\ ${ }^{3}$ LIGO Hanford Observatory, P.O. Box 159 Richland, Washington 99352, USA \\ ${ }^{4}$ California Institute of Technology, 1200 E. California Blvd. Pasadena, California 91125, USA \\ ${ }^{*}$ Corresponding author: keiko@lsu.edu
}

Received September 19, 2013; revised November 8, 2013; accepted November 10, 2013; posted November 11, 2013 (Doc. ID 197967); published December 10, 2013

\begin{abstract}
The effects of residual amplitude modulation (RAM) in laser interferometers using heterodyne sensing can be substantial and difficult to mitigate. In this work, we analyze the effects of RAM on a complex laser interferometer used for gravitational wave detection. The RAM introduces unwanted offsets in the cavity length signals and thereby shifts the operating point of the optical cavities from the nominal point via feedback control. This shift causes variations in the sensing matrix, and leads to degradation in the performance of the precision noise subtraction scheme of the multiple-degree-of-freedom control system. In addition, such detuned optical cavities produce an optomechanical spring, which also perturbs the sensing matrix. We use our simulations to derive requirements on RAM for the Advanced LIGO (aLIGO) detectors, and show that the RAM expected in aLIGO will not limit its sensitivity. (C) 2013 Optical Society of America
\end{abstract}

OCIS codes: $\quad$ (040.0040) Detectors; (120.0120) Instrumentation, measurement, and metrology; (120.5060)

Phase modulation; (140.0140) Lasers and laser optics. http://dx.doi.org/10.1364/JOSAA.31.000081

\section{INTRODUCTION}

The direct detection of gravitational waves (GWs) is a challenging but significant goal for fundamental physics and astronomy in the near future. There are several laser interferometric detector projects around the globe that aim to directly detect GWs from astrophysical sources, such as Advanced

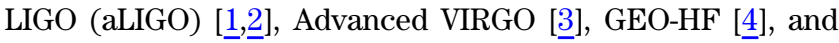
KAGRA [5]

These interferometers are kilometer-scale Michelson interferometers with coupled Fabry-Perot cavities used to enhance the sensitivity to the space-time strain induced by the GWs. The expected strains from astrophysical events should produce displacements of order $10^{-19} \mathrm{~m}$ in these interferometers.

To measure such a small difference with a high signal-tonoise ratio, the lengths of the optical cavities in the interferometer must be controlled by precise positioning of all the constituent optics, ensuring that the optical response to the $\mathrm{GW}$ is linear.

The interferometric length signals are derived by heterodyne detection using a variant of the Pound-Drever-Hall cavity locking technique [6]: the laser light is phase-modulated at a certain frequency before being injected into the interferometer. A signal is then generated by the beat between the fields at different frequencies, such as the carrier field and modulation sidebands. In the aLIGO interferometer, phase modulation (PM) at two different frequencies is introduced to robustly extract the length signals for the multiple degrees of freedom (DoFs).

Due to imperfections (explained in later sections), the electro-optic modulator (EOM) used to impose PM sidebands on the optical field also introduces some residual amplitude modulation (RAM). This RAM is at the same frequencies as the PM and may therefore introduce spurious signals and offsets when the laser fields are sensed by photodetectors and demodulated to generate error signals. This may lead to changes in the frequency response to GWs (i.e., the calibration) and also to spurious couplings of laser noise to the GW readout channel.

In this paper, we present a model of how RAM affects the response of the $\mathrm{GW}$ interferometer and impacts its sensitivity. In Section 2, we introduce RAM and describe its effect on the GW detectors. In Section 3, we give an overview of our simulation setup. Section 4 presents the results: the predicted effect of RAM effect on aLIGO sensitivity. Simulation parameters and details of the sensing and control scheme used in our calculation can be found in Appendix $\underline{A}$ and $\underline{B}$, respectively.

\section{RAM AND ADVANCED GW DETECTORS}

In this section, we first give an overview of the aLIGO optical configuration and its sensing and control scheme. Then, we introduce the issue of RAM and show how it may lead to problems with sensing and control.

A. Optical Configuration and Sensing Scheme of aLIGO Figure 1 shows the optical configuration of aLIGO. Each arm of the interferometer is a 4-km Fabry-Perot cavity that enhances the instrument's response to GW signals. A power recycling cavity (PRC) enhances the effective incident laser power, and a signal recycling cavity (SRC) tunes the detection 


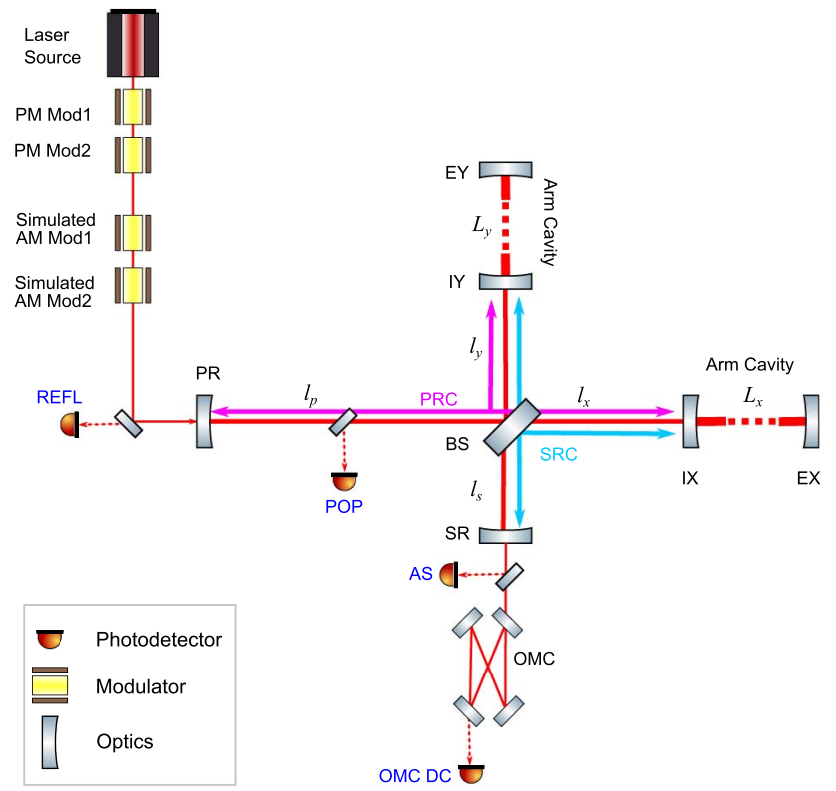

Fig. 1. Full interferometer optical configuration. PR, power recycling mirror; PRC, power recycling cavity; SR, signal recycling mirror; SRC, signal recycling cavity; IX, IY, input test mass at X or Y arm, respectively; EX, EY, end test mass at $\mathrm{X}$ or $\mathrm{Y}$ arm, respectively; $\mathrm{BS}$, beam splitter; REFL, reflection port; AS, antisymetric port; OMC, output mode cleaner; PO, pick-off port; OMC DC, DC readout port at the OMC transmission; PM Mod1 and 2, phase modulators for sideband 1 and 2, respectively; AM Mod1 and 2, amplitude modulators to simulate the RAM. $l_{p}$, PR to BS; $l_{s}$, SR to BS; $l_{x}$ and $l_{y}$, BS to IX and IY, respectively; $L_{x}$ and $L_{y}, x$ and $y$ arm, respectively.

bandwidth. There are five DoFs to control: DARM, the differential motion of the two arm cavities (the GW channel); CARM, the common motion of the arm cavities; MICH, the difference between two short arms of the Michelson interferometer; PRCL, the PRC length, i.e., the average distance between the power-recycling mirror (PRM) and the two input test masses (ITMs, IX and IY in Fig. 1); SRCL, the SRC length, i.e., the average distance between the signal recycling mirror (SRM) to the two ITMs. The explicit definitions of these DoFs are summarized in Table $\underline{1}$.

To robustly extract length signals for the five DoFs, two sets of PM sidebands are used. Mirror motion induces PM of the carrier and sideband laser fields, which experience different resonant conditions in the interferometer due to the careful choice of modulation frequencies. Demodulated signals from photodetectors that sample fields extracted from the interferometer produce signals that contain information about the various DoFs.

\section{B. Electro-Optic Modulator and RAM Source}

An EOM is a device used to modulate the phase of a laser field. An EOM consists of a Pockels cell-a crystal exhibiting a

Table 1. Definitions of the Five Length DoFs to Control

\begin{tabular}{lc}
\hline & Definition \\
\hline DARM & $\left(L_{x}-L_{y}\right) / 2$ \\
CARM & $\left(L_{x}+L_{y}\right) / 2$ \\
MICH & $l_{x}-l_{y}$ \\
PRCL & $l_{p}+\left(l_{x}+l_{y}\right) / 2$ \\
SRCL & $l_{s}+\left(l_{x}+l_{y}\right) / 2$ \\
\hline
\end{tabular}

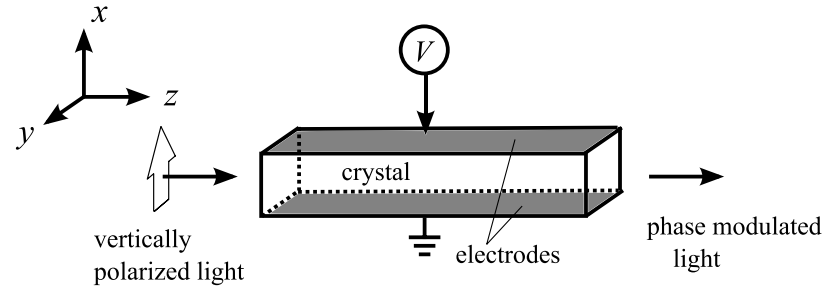

Fig. 2. Electro-optic modulator. The modulation volage $V$ is applied along the crystal in the $x$ axis, which is one of the crystal principal axes. The polarized field in the $x$ axis is phase-modulated after passing through the crystal. The beam propagates in the $z$ direction.

birefringence that depends linearly on the applied electric field-and a set of electrodes. The electrodes, attached on the top and bottom surfaces of the crystal, apply a voltage along one of the principal axes of the crystal. Figure 2 shows the EOM crystal with voltage applied along the $x$ axis, and the laser light polarized along the $x$ axis as an input. As the laser light passes through the crystal, the birefringence of the crystal changes due to the externally applied electric field and the laser light is phase-shifted. The phase shift induced by the electric field is written as $\Delta \phi=\pi n_{x}^{3} r V / \lambda$, where $n_{x}, r, V, \lambda$ are the unperturbed refractive index in the $x$ direction, an electro-optic coefficient, the applied voltage, and the laser wavelength, respectively [7].

During the PM process, unwanted amplitude modulation is also imposed due to the following effects [8-10], which are difficult to avoid in practice: (1) Axis mismatch between the incident polarization and the crystal orientation. When the input beam polarization axis does not align with one of the crystal axes and the axis of the applied electric field, the projections of the input field onto the crystal's orthogonal axes obtain different phase shifts. For example, in Fig. 2, when the beam polarization is not along the $x$ direction, the laser field polarized in the $x$ direction obtains a phase shift, while the laser field polarized in the $y$ direction obtains a different phase shift. This leads to an effective rotation of the transmitted beam's polarization, which, upon its subsequent interaction with polarizing optics, leads to AM. (2) Etalons in the crystal. Due to the finite reflectivities of the crystal faces, some light is circulated within the crystal. The multipass light field experiences a frequency-dependent phase shift and amplitude envelope due to this etalon effect.

In practice, the use of good antireflection coatings and wedged crystal faces makes (1) the dominant contribution to RAM.

Whatever the coupling, temperature fluctuations are usually the major driving force of RAM generation. Temperature drifts affect the mounting orientation of the crystal through the thermal expansion of the mounting material, and also lead directly to expansion of the crystal and modulation of its static birefringence. As such, a first approach to reducing RAM is simple temperature stabilization of the crystal and enclosure. More sophisticated techniques involve measuring the RAM optically and actively feeding back to either the modulation voltage [9] or the crystal temperature [11].

\section{Residual Amplitude Modulation}

RAM is imposed at the same frequency as that of the intentional PM. A laser field whose phase and amplitude are modulated at a frequency $\omega_{m}$ can be expressed as 


$$
E=E_{\text {in }}\left[1+\Gamma_{a} \sin \left(\omega_{m} t+\phi\right)\right] \times \exp \left[i \omega_{0} t+i \Gamma_{p} \sin \omega_{m} t\right]
$$

where $E_{\text {in }}$ is the amplitude of the field, $\omega_{0}$ denotes the angular frequency of the carrier field, $\Gamma_{a}$ and $\Gamma_{p}$ are the modulation depths of the RAM and PM, respectively, and $\phi$ is the relative phase between the RAM and PM terms. In order to evaluate the amount of the RAM relative to the PM, we define the RAM-to-PM ratio as

$$
\eta \equiv \frac{\Gamma_{a}}{\Gamma_{p}}
$$

When PM is imposed at two different frequencies, the field with RAM can be written as

$$
\begin{aligned}
E= & E_{\text {in }}\left[1+\Gamma_{a 1} \sin \left(\omega_{m 1} t+\phi_{A 1}\right)+\Gamma_{a 2} \sin \left(\omega_{m 2} t+\phi_{A 2}\right)\right] \\
& \times \exp \left[i \omega_{0} t+i \Gamma_{p 1} \sin \left(\omega_{m 1} t+\phi_{P 1}\right)\right. \\
& \left.+i \Gamma_{p 2} \sin \left(\omega_{m 2} t+\phi_{P 2}\right)\right] .
\end{aligned}
$$

where $\phi_{A 1}, \phi_{A 2}, \phi_{P 1}$, and $\phi_{P 1}$ are the arbitrary phases of the RAM and PM terms.

Since they are at the same frequencies as the PM sidebands, the RAM sidebands introduce unwanted signal offsets $[\underline{8}, \underline{12}]$ when interferometer signals for a given DoF are extracted from the beat between the carrier field and a control sideband on a photodetector. Because the interferometer optics' positions are controlled by servoing these error signals to zero, the offsets due to RAM can change their positions, affecting the interferometer response and degrading its sensitivity to GWs.

\section{SIMULATIONS}

To evaluate the effect of RAM on the GW sensitivity, we used a frequency-domain simulation tool, Optickle $[\underline{13}, \underline{14}]$. The aLIGO optical configuration (shown in Fig. 1) was modeled with parameters summarized in Appendix A.

There are three signal extraction ports in the interferometer: reflection (REFL) port, antisymmetric (AS) port, and pick-off port (POP, pick-off field from PRC). One sensor (photodiode) at a signal extraction port is assigned for each DoF so that the sensor has the maximum sensitivity to the assigned DoF. Similarly, an output matrix feeds the derived control signals to an optic position actuation path in the right column.

The GW channel (DARM) is detected at the output mode cleaner transmission port (OMC DC). This "DC readout" is a homodyne detection method, which requires the introduction of a small DARM offset to bring the AS port slightly off of a dark fringe (producing linear sensitivity to the DARM DoF). This scheme is proposed for the GW readout due to its better signal-to-noise ratio compared with the heterodyne readout [15-17].

The actuation mapping on the right-hand side of Table $\underline{2}$ is chosen so as to maintain the orthogonality of the DoFs as best as possible; in practice, there is always some cross coupling between DoFs. In the case of aLIGO, DARM is polluted most seriously by MICH and SRCL. To mitigate this effect, feedforward is applied from these DoFs to DARM-this feature is included in our simulation. See Appendix $\underline{B}$ for more information about the sensing and control scheme.
Table 2. Input-Output Chain for aLIGO Setup $^{a}$

\begin{tabular}{lcc}
\hline DoF & Input & Output \\
\hline DARM & OMC DC & EX - EY \\
CARM & REFL f1 I & EX + EY \\
MICH & REFL f2 Q & $\sqrt{2} \mathrm{BS}-\mathrm{PR}+\mathrm{SR}$ \\
PRCL & REFL f2 I & PR \\
SRCL & POP f2 I & SR \\
\hline
\end{tabular}

${ }^{a}$ The input column shows the sensors used to detect each DoF with I (I-phase) or Q (Q-phase) demodulation phase. f1 and f2 represent the demodulation frequency. The output column shows which optics are fed back to for control of each DoF.

The frequency dependence from force to displacement of the suspended test masses is approximated using a transfer function where the response is flat up to $1 \mathrm{~Hz}$ peak and then drops as $f^{-2}$. This is an approximation of the force-todisplacement transfer function for the test mass stage in the quadruple suspensions. The input and end test masses (therefore, the Fabry-Perot arm cavities) are suspended by the quadruple suspensions in aLIGO [1].

\section{SIMULATION RESULTS}

\section{A. Operating Point Offsets}

The length offsets of each DoF due to RAM are shown in Fig. 3 . These were calculated by the following iterative process: first, the error signal offset due to RAM is calculated. Then, the operating point of each DoF is changed so that the error signals are zero for the corresponding DoF. The error signal now has the different RAM offsets because the interferometer response is slightly changed by the length offsets. Therefore, a new length offset is added to make the new error signal zero. We iterate this process until the RAM offset and the operation point converge to below $10^{-12}$ times the laser wavelength. RAM was applied at both modulation frequencies.

The largest effect of RAM is on the SRCL DoF, with smaller effects seen on PRCL and MICH. We can also see that a larger RAM level produces larger length offsets. CARM and DARM (EX and EY in Fig. 3) are less sensitive to RAM because the

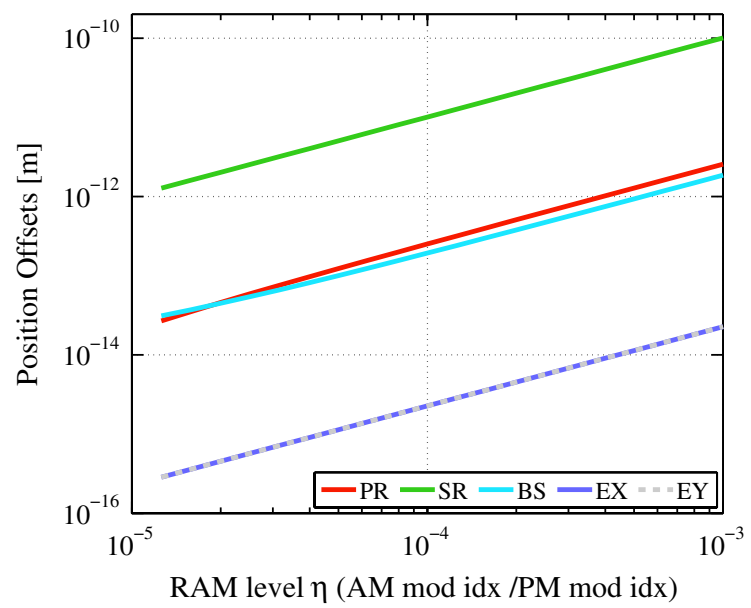

Fig. 3. Length offsets on each optic to make the length error signal for each DoF zero, as a function of the RAM level. Larger RAM adds more offsets on the error signals, resulting in the length offsets of the optics. 
frequency discriminants of the corresponding error signals are enhanced by the Fabry-Perot arm cavities. In addition, the DARM DoF is read out using homodyne detection (i.e., there are no RF sidebands) and is therefore only sensitive to RAM via the cross-coupling effects described above.

\section{B. Effects on Optomechanical Response}

The optomechanical compliance is altered when RAM is present. Figure $\underline{4}$ shows the transfer function from force to displacement of the test mass stage on the quadruple suspension (EX). The transfer functions with $\eta=0-10^{-2}$ are depicted. Optomechanical peaks appear even when RAM is absent, $\eta=0$, because of the slight DARM offset for DC readout ( $3 \mathrm{pm}$ is used here). With $\eta \gtrsim 10^{-4}$ or more, the optomechanical peaks become sharper due to the length offsets shown in the previous subsection. These small offsetsparticularly those in SRCL-result in detuned operation of the SRC $\left(0.08 \mathrm{deg}\right.$ for $\left.\eta=10^{-3}\right)$, which increases the effect of the optical spring in the coupled DARM-SRCL cavity [19]. The modification of the optomechanical response in these complicated ways requires careful tailoring of the feedback controller in order to maintain the same level optimal control. Reduction of the low-frequency gain can increase upconversion due to nonlinearity of the optical error signal and reduction of the phase/gain margins around the unity gain frequency of the loop reduce the robustness of the loop and increase signal calibration variation, which can compromise astrophysical signal detection [20,21] and parameter estimation [22].

\section{Strain Sensitivity and Loop Noise}

Due to this change in optical response, the resulting strain sensitivity is altered as shown in Fig. 5 . There is no significant
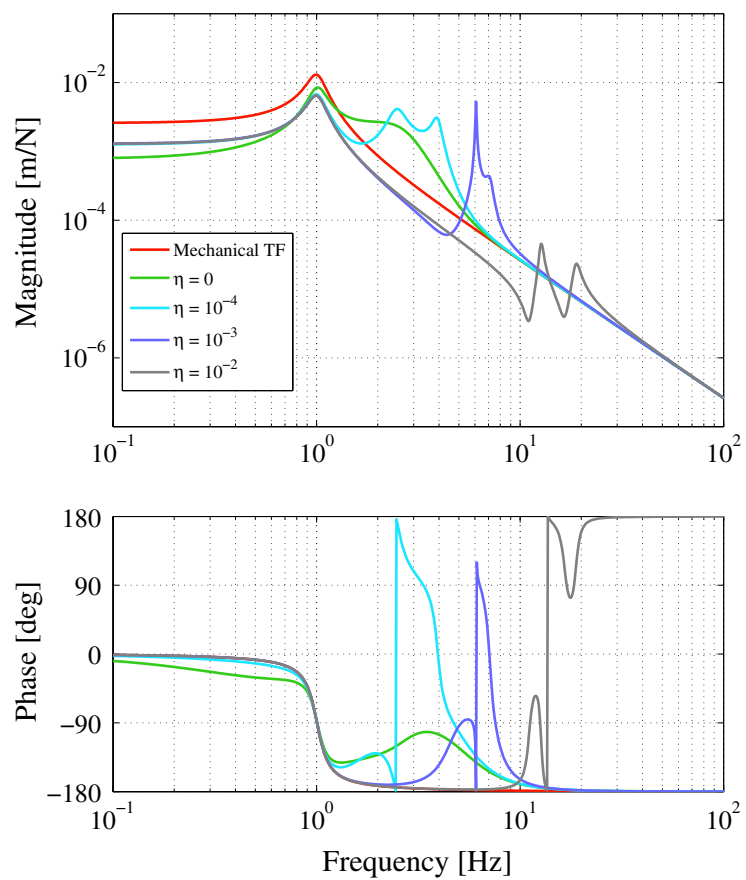

Fig. 4. Mechanical transfer function from the force to displacement of the suspension $(\mathrm{EX})$ in $[\mathrm{m} / \mathrm{N}]$ (red) and the modified optomechanical transfer functions with various RAM-to-PM ratios and DARM offset for DC readout. Length offsets produce detuning of the interferometer's coupled optical cavities.

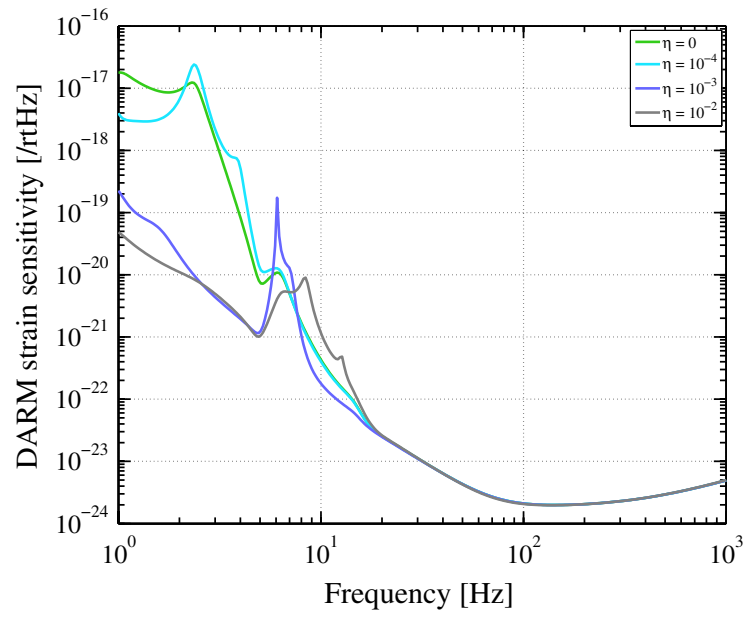

Fig. 5. Quantum-noise-limited DARM sensitivity with $\eta=0,10^{-4}$, $10^{-3}$, and $10^{-2}$. The intrinsic quantum noise of DARM and the quantum noise contributions (i.e., the noise at each sensor propagated through to each control signal) from auxiliary DoFs are considered. The sensitivity at low frequencies is limited by the cross-coupled noise from auxiliary DoFs (see, Fig. 6 for the breakdown of the cross couplings). The cross-coupled noise shape was changed due to the length offsets generated by RAM.

difference with $\eta \approx 10^{-4}$, the level measured in the EOM in use at the LIGO Livingston Observatory. Even with larger $\eta$, the quantum noise is little affected above $10 \mathrm{~Hz}$.

Note that quantum noise contributions from auxiliary DoFs are included in Fig. 5, in addition to the quantum noise intrinsic to DARM. In Fig. 6 , we see the breakdown of the DARM sensitivity for the case of $\eta=10^{-3}$. The noise intrinsic to the DARM loop is shown in the red trace, and all other traces are cross couplings. Below $\sim 12 \mathrm{~Hz}$, the total noise (i.e., the black trace in Fig. 6) is dominated by contributions from other DoFs. This is the reason for the seeming enhancement in DARM sensitivity with increasing RAM; the contribution from cross coupling is indeed suppressed due to the modified response, but the noise intrinsic to the DARM loop experiences no such reduction. Therefore, the ideal quantum-noise-limited

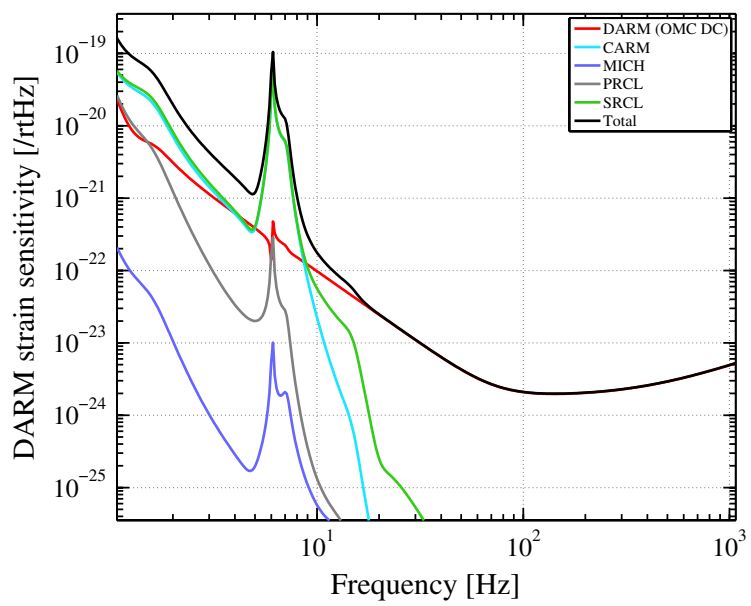

Fig. 6. Loop noise picture of DARM when $\eta=10^{-3}$. Shown here is the DARM sensing noise (OMC_DC), as well as the cross-coupled control noise from each auxiliary DoF. The optical spring appears through the loop noise mixing while the OMC_DC noise floor is slightly changed by RAM. The low-frequency interferometer performance is limited mainly by the SRCL control noise in our control loop model. 
GW sensitivity is not improved by the introduction of RAM. To the contrary, it is diminished for higher levels of RAM, and it is not effected appreciably by the levels considered here. In any case, the sensitivity of aLIGO to GWs is predicted to be completely dominated by seismic noise and suspension thermal noise at these low frequencies.

\section{CONCLUSIONS}

We have studied the effect of RAM on the sensitivity of advanced laser-interferometric GW detectors using numerical, frequency-domain simulation. As our main result, we have found that a realistic expected RAM-to-PM ratio of $10^{-4}$ (measured at aLIGO Livingston) does not pose a threat to GW sensitivity in the case of aLIGO. However, while also not appreciably affecting sensitivity, we have shown that RAM levels modestly exceeding this can have pronounced effects on the optomechanical response of the interferometer. Therefore, care should be taken to minimize RAM so as to avoid potential instabilities.

\section{APPENDIX A: SIMULATION PARAMETERS}

The optical parameters are chosen to model the aLIGO Livingston interferometer, as listed in Table $\underline{3}$ and found in [23,24]. The servo filters are chosen to have a unity gain frequency of $200 \mathrm{~Hz}$ for DARM, $50 \mathrm{kHz}$ for CARM, and $20 \mathrm{~Hz}$ for PRCL, $15 \mathrm{~Hz}$ for $\mathrm{MICH}$ and $10 \mathrm{~Hz}$ for SRCL.

\section{APPENDIX B: SENSING SCHEME}

In this section, we describe in detail the sensing signals and the control scheme used in our simulation, including a brief description of the multiple-DoF control method.

\section{Sensing and Control}

Figure 7 is the radar plot representing the signals in each sensor. The five DoF signals in each sensor (i.e., in each radar) are shown as arrows. The arrow length is the signal strength in $\mathrm{W} / \mathrm{m}$ in logarithmic scale and the angles are the optimum demodulation phases in degrees. Because the DC readout

Table 3. aLIGO Parameters Used in Our Simulation ${ }^{a}$

\begin{tabular}{|c|c|}
\hline Arm lengths & $3994.5 \mathrm{~m}$ \\
\hline Arm cavity FWHM/FSR & $84.2344 \mathrm{~Hz} / 37.526 \mathrm{kHz}$ \\
\hline PRC length & $57.6562 \mathrm{~m}$ \\
\hline PRC FWHM/FSR & $13.190 \mathrm{kHz} / 2.5998 \mathrm{MHz}$ \\
\hline SRC length & $56.0082 \mathrm{~m}$ \\
\hline SRC FWHM/FSR & $179.18 \mathrm{kHz} / 3.7526 \mathrm{MHz}$ \\
\hline Schnupp Asymmetry & $8 \mathrm{~cm}$ \\
\hline Short Michelson arm length & $5.3428 \mathrm{~m}$ (average) \\
\hline PRM transmissivity & 0.03 \\
\hline SRM transmissivity & 0.35 \\
\hline ITM transmissivity & 0.14 \\
\hline ETM transmissivity & $5 \mathrm{ppm}$ \\
\hline Input laser power & $135 \mathrm{~W}$ or $25 \mathrm{~W}$ \\
\hline Optics loss (per mirror) & $30 \mathrm{ppm}$ \\
\hline OMC transmissivity & 0.99 \\
\hline PM1 frequency & $9.099471 \mathrm{MHz}$ \\
\hline PM2 frequency & $45.497355 \mathrm{MHz}$ \\
\hline PM1 modulation index & 0.1 \\
\hline PM2 modulation index & 0.1 \\
\hline
\end{tabular}

${ }^{a} \mathrm{FWHM}$, the full width at half-maximum; FSR, free spectral range. signal (OMC DC sensor) for DARM is a DC signal and does not have demodulation phase, it is not listed in the radar plot.

The desired signal at each sensor is obtained by demodulating at the optimum demodulation phase for the targeted DoF. Undesired signals mix into the obtained signal with a ratio determined by the relative phase separation from the desired signal. The sensors are chosen to, as much as possible, separate the various length signals from one another to maximize sensitivity to the desired DoF and minimize cross coupling from other DoFs. The signals shown in Fig. 7 are calculated at $20 \mathrm{~Hz}$, with an input power of $25 \mathrm{~W}$, and a differential arm offset of $3 \mathrm{pm}$.

Table $\underline{4}$ shows the conventional sensing matrix of our model. This matrix is often used to express the signal sensitivity to the different DoFs, at each detection port, at a certain frequency [25]. The first column of a sensing matrix shows the sensors, which is a choice of the detection port, demodulation frequency, and demodulation phase (I- or Q-phase), and the rows of a sensing matrix show the gains of the optical response for each DoF. The diagonal elements of the matrix correspond to the sensitivity of the given sensor to the DoF to be controlled by it, and the off-diagonal elements show the contributions from the other DoFs. One sensor is fed back to one DoF for the control, therefore, we have five sensors to control the five DoFs.

For example, in our model, OMC DC is chosen for the DARM control, having the maximum sensitivity to DARM, with some contribution from PRCL, MICH, and SRCL.

\section{Signal Mixture}

The fact that each sensor is sensitive to all DoFs (in other words, the sensing matrix is not completely diagonal), leads to difficulties with interferometer sensing and control. When the sensor signal at a given port is fed back to its target interferometer DoF, some non-target-DoF signal is also fed back to that DoF due to these cross couplings.

The DARM signal at the antisymmetric ports (AS and OMC DC) cannot be cleanly separated from that of MICH becausefrom the point of view of the dark port-differential arm or Michelson motion are essentially equivalent (the gain at the AS port is much larger for DARM than for MICH because DARM is enhanced by the arm cavities). Therefore, there is always a MICH signal mixing into the DARM feedback signal, even if the displacement in the MICH DoF is suppressed to the shot-noise level: Because the optical gain of MICH is smaller than that of DARM, which is enhanced by the resonant arm cavity, the shot-noise-limited sensitivity of MICH is worse than DARM. As a consequence, the cross-coupled MICH signal increases the effective shot noise of the DARM sensor.

As for second-order effects, the SRCL, PRCL, and CARM signals are also mixed into DARM through the MICH mixture path [26]. The SRCL and PRCL sensors have higher shot noise level than that of DARM, because they are (in general) extracted at POP or REFL, both of which have higher light power than the DARM port at the dark fringe. Also, SRCL is almost always degenerate with the other DoFs and difficult to extract independently because the finesse of SRC is much lower than those of the other DoFs. Therefore, SRCL has a relatively low signal-to-noise ratio, and the shot noise at the SRCL sensor appears in the displacement sensitivity of DARM by way of the MICH mixture path. 

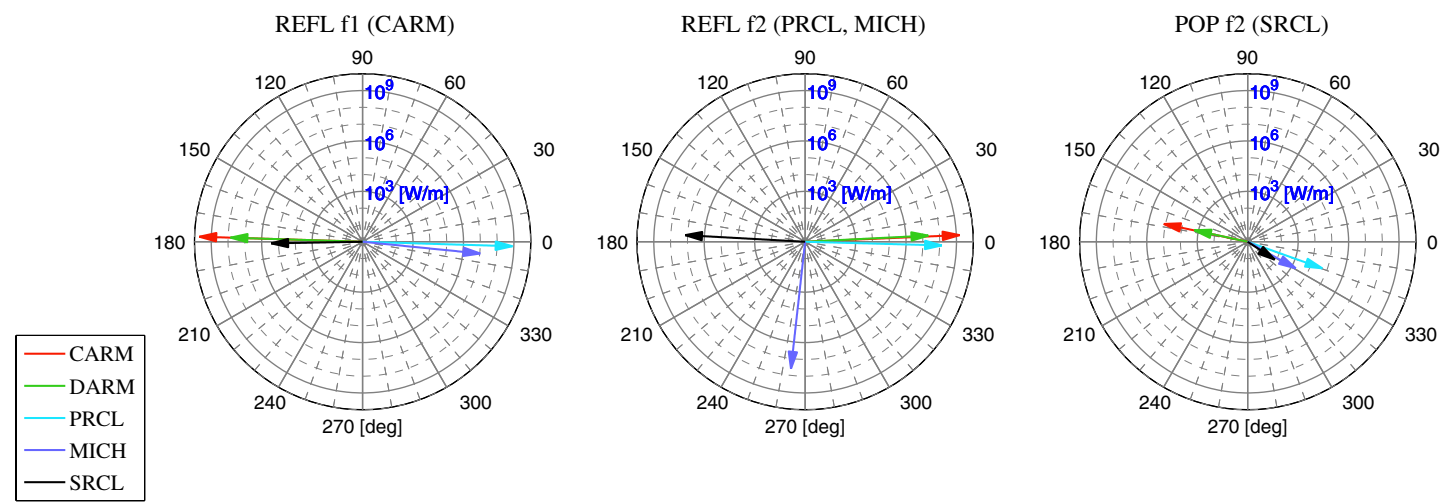

Fig. 7. Radar representation of the sensing matrix. The arrow lengths represent the signal strength [W/m], and the angles show the demodulation phases for each signal. OMC DC sensor for DARM is not shown because it is a DC readout signal and does not have demodulation phase.

Table 4. Sensing Matrix ${ }^{a}$

\begin{tabular}{lccrrrr}
\hline Sensor & Phase & CARM & DARM & PRCL & MICH & SRCL \\
\hline REFL I1 & $178^{\circ}$ & $5.10 \times 10^{9}$ & $7.16 \times 10^{7}$ & $-8.23 \times 10^{8}$ & $-1.08 \times 10^{7}$ & $2.67 \times 10^{5}$ \\
OMC DC & N/A & $6.63 \times 10^{0}$ & $1.77 \times 10^{5}$ & $1.14 \times 10^{3}$ & $6.32 \times 10^{1}$ \\
REFL I2 & $-2^{\circ}$ & $1.54 \times 10^{9}$ & $2.15 \times 10^{7}$ & $1.36 \times 10^{8}$ & $-3.35 \times 10^{6}$ & $-1.39 \times 10^{2}$ \\
REFL Q2 & $-96^{\circ}$ & $-2.37 \times 10^{8}$ & $-3.46 \times 10^{6}$ & $-1.15 \times 10^{7}$ & $3.97 \times 10^{7}$ & $7.85 \times 10^{5}$ \\
POP I2 & $34^{\circ}$ & $-1.18 \times 10^{5}$ & $-1.66 \times 10^{3}$ & $4.92 \times 10^{4}$ & $1.84 \times 10^{3}$ \\
\hline
\end{tabular}

${ }^{a} \mathrm{DC}$ readout offset (DARM DC offset) of $3 \mathrm{pm}$ is assumed. They are optical gains [W/m] at $20 \mathrm{~Hz}$. Demodulation phases are optimized for the diagonal elements in each sensor.

Figure $\underline{8}$ shows a simplified diagram of the control loops. For example, shot noise is added at each sensor into its respective loop, and coupled into the DARM error signal. This coupling is shown as the control noises in Fig. $\underline{6}$ by the following process:

$$
\vec{V}_{\mathrm{errC}}=(1-\mathbb{G})^{-1} \mathbb{I M A O} \vec{V}_{\mathrm{ctrl}},
$$

where $\vec{V}_{\text {ctrl }}$ is the control signal vector for the five DoFs, 1 is an identity matrix, $\mathbb{G}$ is the open-loop transfer function (in the frequency domain) of the five DoF loop including the servo filter shapes, $\mathbb{F}$, actuator matrix, $\mathbb{A}$, input matrix, $\mathbb{I}$, output matrix, $\mathbb{O}$, and the optical response as a matrix, $\mathbb{M}$. Input and output matrices are the matrices to change either from the sensor basis to the DoF basis, or, from the DoF basis to the actuator basis, respectively (i.e., the matrix form of Table 2). Here, $\mathbb{G}, \mathbb{M}, \mathbb{I}$, and $\mathbb{O}$ are $5 \times 5$ matrices and $\mathbb{F}$ and $\mathbb{A}$ are $5 \times 5$ diagonal matrices. Note that the radiation pressure is also summed into the quantum noises in Fig. 6 .

The calibrated displacement sensitivity $d \vec{L}$ is written as

$$
d \vec{L}=\mathbb{M}^{-1}(1+\mathbb{G}) \vec{V}_{\mathrm{err}} .
$$

As neither $\mathbb{G}$ nor $\mathbb{M}$ is diagonal, shot noise at every sensor pollutes the DARM sensitivity. The optical cross-coupling path is shown as gray dashed line on the bottom left in Fig. 8 .

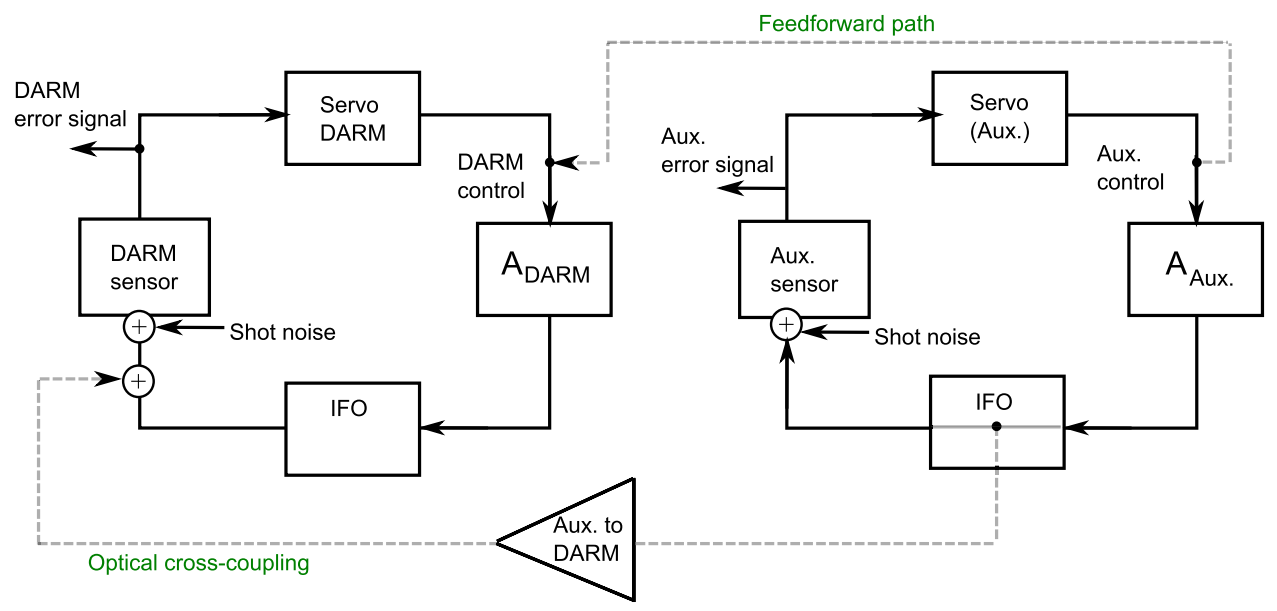

Fig. 8. Diagram of the signal mixture from an auxiliary to the DARM loop. Shot noise in the CARM, PRCL, MICH, and SRCL (shown as "Aux. DoF") are added at each sensor, and are mixed into the DARM loop through the nondiagonal interferometer (IFO) response (lower dashed arrow). The feedforward path in the simulation works between the DARM control signal and control signal of the auxiliary DoFs, as shown in the gray dashed trace on upper right. "A" is the actuator for DARM or Aux. 


\section{Calibration}

To calibrate the DARM displacement, $d L_{\mathrm{DARM}}[\mathrm{m} / \sqrt{\mathrm{Hz}}]$ [the DARM element in Eq. (B2)], into strain sensitivity, $h[1 / \sqrt{\mathrm{Hz}}]$, down to low frequencies, we have to take into account the suspension transfer function of the test masses (or optomechanical transfer function when optical spring effect gives rise). Treating the $\mathrm{GW}$ as tidal force, we have

$$
d L_{\mathrm{DARM}}=-\frac{d L}{d F} m \omega^{2} h L,
$$

where $L$ is the arm length, $\omega$ is the angular frequency of the signal, $(d L / d F)$ is the (opto-) mechanical transfer function. Note that Eq. (B3) is valid where the GW length is larger than $L$. In our case of $L \sim 4 \mathrm{~km}$, this calibration method is valid from $\mathrm{DC}$ to $75 \mathrm{kHz}$ [27].

\section{Feedforward}

A feedforward technique is used to partially suppress the coupling from the auxiliary DoF control signals into the DARM control signal. As evidenced by the off-diagonal elements of the sensing matrix, there are cross couplings from all the auxiliary DoFs into the DARM. In particular the SRCL signal cannot be extracted independently, and it creates a large second-order coupling. In our simulation, the couplings from SRCL to DARM, and from MICH to DARM are subtracted from the control signal by the feedforward path (dashed gray line in Fig. 8) so as to subtract the undesired contributions from SRCL and MICH above $20 \mathrm{~Hz}$. The suppression ratios due to the feedforward at $20 \mathrm{~Hz}$ are $0.4 \%$ for $\mathrm{MICH}$ and $4 \%$ SRCL control noise. They were not degraded by the presence of RAM.

This feedforward method was used in the first-generation GW detector Initial LIGO and is planned to be implemented in aLIGO, as well.

\section{ACKNOWLEDGMENTS}

We thank the reviewers for their detailed and perceptive commentary. We would like to thank Peter Fritschel and Gabriela Gonzalez for their comments on an early version of the manuscript and our colleagues at the LIGO Livingston Observatory for sharing with us their experiences with RAM over long periods of time. KK was supported by the National Science Foundation with awards 0905184 and 1205882. We also gratefully acknowledge the National Science Foundation for their support under grant PHY-0555406.

\section{REFERENCES}

1. LIGO Scientific Collaboration, "LIGO: the laser interferometer gravitational-wave observatory,” Rep. Prog. Phys. 72, 076901 (2009).

2. G. M. Harry, and the LIGO Scientific Collaboration, "Advanced LIGO: the next generation of gravitational wave detectors," Classical Quantum Gravity 27, 084006 (2010).

3. F. Acernese, P. Amico, M. Alshourbagy, F. Antonucci, S. Aoudia, S. Avino, D. Babusci, G. Ballardin, F. Barone, L. Barsotti, M. Barsuglia, F. Beauville, S. Bigotta, S. Birindelli, M. A. Bizouard, C. Boccara, F. Bondu, L. Bosi, C. Bradaschia, S. Braccini, A. Brillet, V. Brisson, L. Brocco, D. Buskulic, E. Calloni, E. Campagna, F. Cavalier, R. Cavalieri, G. Cella, E. Cesarini, E. Chassande-Mottin, C. Corda, F. Cottone, A.-C. Clapson, F. Cleva, J.-P. Coulon, E. Cuoco, A. Dari, V. Dattilo, M. Davier, R. D. Rosa, L. D. Fiore, A. D. Virgilio, B. Dujardin, A. Eleuteri D. Enard, I. Ferrante, F. Fidecaro, I. Fiori, R. Flaminio, J.-D.
Fournier, O. Francois, S. Frasca, F. Frasconi, A. Freise, L. Gammaitoni, F. Garufi, A. Gennai, A. Giazotto, G. Giordano, L. Giordano, R. Gouaty, D. Grosjean, G. Guidi, S. Hebri, H. Heitmann, P. Hello, L. Holloway, S. Karkar, S. Kreckelbergh, P. L. Penna, M. Laval, N. Leroy, N. Letendre, M. Lorenzini, V. Loriette, M. Loupias, G. Losurdo, J.-M. Mackowski, E. Majorana, C. N. Man, M. Mantovani, F. Marchesoni, F. Marion, J. Marque, F. Martelli, A. Masserot, M. Mazzoni, L. Milano, C. Moins, J. Moreau, N. Morgado, B. Mours, A. Pai, C. Palomba, F. Paoletti, S. Pardi, A. Pasqualetti, R. Passaquieti, D. Passuello, B. Perniola, F. Piergiovanni, L. Pinard, R. Poggiani, M. Punturo, P. Puppo, K. Qipiani, P. Rapagnani, V. Reita, A. Remillieux, F. Ricci, I. Ricciardi, P. Ruggi, G. Russo, S. Solimeno, A. Spallicci, R. Stanga, R. Taddei, M. Tonelli, A. Toncelli, E. Tournefier, F. Travasso, G. Vajente, D. Verkindt, F. Vetrano, A. Viceré, J.-Y. Vinet, H. Vocca, M. Yvert, and Z. Zhang, "The Virgo status," Classical Quantum Gravity 23, S635 (2006).

4. H. Lück, M. Hewitson, P. Ajith, B. Allen, P. Aufmuth, C. Aulbert, S. Babak, R. Balasubramanian, B. W. Barr, S. Berukoff, A. Bunkowski, G. Cagnoli, C. A. Cantley, M. M. Casey, S. Chelkowski, Y. Chen, D. Churches, T. Cokelaer, C. N. Colacino, D. R. M. Crooks, C. Cutler, K. Danzmann, R. J. Dupuis, E. Elliffe, C. Fallnich, A. Franzen, A. Freise, I. Gholami, S. Goßler, A. Grant, H. Grote, S. Grunewald, J. Harms, B. Hage, G. Heinzel, I. S. Heng, A. Hepstonstall, M. Heurs, S. Hild, J. Hough, Y. Itoh, G. Jones, R. Jones, S. H. Huttner, K. Kötter, B. Krishnan, P. Kwee, M. Luna, B. Machenschalk, M. Malec, R. A. Mercer, T. Meier, C. Messenger, S. Mohanty, K. Mossavi, S. Mukherjee, P. Murray, G. P. Newton, M. A. Papa, M. Perreur-Lloyd, M. Pitkin, M. V. Plissi, R. Prix, V. Quetschke, V. Re, T. Regimbau, H. Rehbein, S. Reid, L. Ribichini, D. I. Robertson, N. A. Robertson, C. Robinson, J. D. Romano, S. Rowan, A. Rüdiger, B. S. Sathyaprakash, R. Schilling, R. Schnabel, B. F. Schutz, F. Seifert, A. M. Sintes, J. R. Smith, P. H. Sneddon, K. A. Strain, I. Taylor, R. Taylor, A. Thüring, C. Ungarelli, H. Vahlbruch, A. Vecchio, J. Veitch, H. Ward, U. Weiland, H. Welling, L. Wen, P. Williams, B. Willke, W. Winkler, G. Woan, and R. Zhu, "Status of the GEO600 detector," Classical Quantum Gravity 23, S71 (2006).

5. K. Somiya, "Detector configuration of KAGRA-the Japanese cryogenic gravitational-wave detector," Classical Quantum Gravity 29, 124007 (2012).

6. P. Fritschel, R. Bork, G. González, N. Mavalvala, D. Ouimette, H. Rong, D. Sigg, and M. Zucker, "Readout and control of a powerrecycled interferometric gravitational-wave antenna," Appl. Opt. 40, 4988-4998 (2001).

7. M. Bass, C. DeCusatis, J. Enoch, V. Lakshminarayanan, G. Li, C. MacDonald, V. Mahajan, and E. Van Stryland, Handbook of Optics, Third Edition Volume II: Design, Fabrication and Testing, Sources and Detectors, Radiometry and Photometry (McGraw-Hill Professional, 2009).

8. E. A. Whittaker, M. Gehrtz, and G. C. Bjorklund, "Residual amplitude modulation in laser electro-optic phase modulation," J. Opt. Soc. Am. B 2, 1320-1326 (1985).

9. N. C. Wong and J. L. Hall, "Servo control of amplitude modulation in frequency-modulation spectroscopy: demonstration of shotnoise-limited detection,” J. Opt. Soc. Am. B 2, 1527-1533 (1985).

10. Newport Corp., High-Frequency Electro-Optic Phase Modulators -Model Series 44xx and 485xx User's Manual.

11. L. Li, F. Liu, C. Wang, and L. Chen, "Measurement and control of residual amplitude modulation in optical phase modulation," Rev. Sci. Instrum. 83, 043111 (2012).

12. W. Wu, "Instrumentation of the next generation gravitational wave detector: triple pendulum suspension and electro-optic modulator," Ph.D. thesis (University of Florida, 2007).

13. M. Evans, "Optickle,"http://www.ligo.mit.edu/ mevans/resources. html.

14. M. Evans, “Optickle," Tech. Rep. T070260-v1 (Massachusetts Institute of Technology, 2007).

15. R. L. Ward, "Length sensing and control of a prototype advanced interferometric gravitational wave detector," Ph.D. thesis (California Institute of Technology, 2010).

16. T. T. Fricke, "Homodyne detection for laser-interferometric gravitational wave detectors," Ph.D. thesis (Louisiana State University, 2011) 
17. A. Buonanno, Y. Chen, and N. Mavalvala, "Quantum noise in laser-interferometer gravitational-wave detectors with a heterodyne readout scheme," Phys. Rev. D 67, 122005 (2003).

18. S. M. Aston, M. A. Barton, A. S. Bell, N. Beveridge, B. Bland, A. J. Brummitt, G. Cagnoli, C. A. Cantley, L. Carbone, A. V. Cumming, L. Cunningham, R. M. Cutler, R. J. S. Greenhalgh, G. D. Hammond, K. Haughian, T. M. Hayler, A. Heptonstall, J. Heefner, D. Hoyland, J. Hough, R. Jones, J. S. Kissel, R. Kumar, N. A Lockerbie, D. Lodhia, I. W. Martin, P. G. Murray, J. O’Dell, M. V. Plissi, S. Reid, J. Romie, N. A. Robertson, S. Rowan, B. Shapiro, C. C. Speake, K. A. Strain, K. V. Tokmakov, C. Torrie, A. A. van Veggel, A. Vecchio, and I. Wilmut, "Update on quadruple suspension design for advanced LIGO," Classical Quantum Gravity 29, 235004 (2012).

19. A. Buonanno and Y. Chen, "Signal recycled laser-interferometer gravitational-wave detectors as optical springs," Phys. Rev. D 65, 042001 (2002).

20. LIGO Scientific Collaboration, "Calibration of the LIGO gravitational wave detectors in the fifth science run," Nucl. Instrum. Methods Phys. Res. Sect. A 624, 223-240 (2010).
21. L. Lindblom, "Optimal calibration accuracy for gravitationalwave detectors," Phys. Rev. D 80, 042005 (2009).

22. N. Silver, The Signal and the Noise: Why So Many Predictions Fail-But Some Don't (Penguin Group US, 2012).

23. R. Abbott, R. X. Adhikari, S. Ballmer, L. Barsotti, M. Evans, P. Fritschel, V. Frolov, G. Mueller, B. Slagmolen, and S. Waldman, "AdvLIGO interferometer sensing and control conceptual design," Tech. Rep. LIGO-T070247 (2007).

24. M. E. L. Barsotti and P. Fritschel, "Advanced LIGO ISC design study for low power operation," Tech. Rep. T1200128-v4 (2012).

25. S. Sato, S. Kawamura, K. Kokeyama, F. Kawazoe, and K. Somiya, "Diagonalization of the length sensing matrix of a dual recycled laser interferometer gravitational wave antenna," Phys. Rev. D 75, 082004 (2007).

26. K. Somiya and O. Miyakawa, "Shot-noise-limited control-loop noise in an interferometer with multiple degrees of freedom," Appl. Opt. 49, 4335-4342 (2010).

27. P. R. Saulson, Fundamentals of Interferometric Gravitational Wave Detectors (World Scientific, 1994). 